Hypothesis

\title{
Mitochondrial genome: defects, disease, and evolution
}

\author{
Angus Clarke
}

\begin{abstract}
Defects of mitochondrial function are often caused by defects of the mitochondrial genome. The hypothesis that defective organelles may spread through syncytial tissues as a result of a process of subcellular Darwinian selection is proposed. Tissues are likely to be involved in mitochondrial disease if they are syncytial, are derived from a few embryonic cells only, have little redundancy of function, and are subject to repeated metabolic stress. These effects, together with the random distribution of genetically heterogeneous mitochondria within the fertilised zygote, may account for the varied clinical pictures of mitochondrial disease. Evolution will have favoured the shift of mitochondrial DNA sequences to the nucleus, once the differentiation of tissues had created body compartments in which defective mitochondria could flourish to the detriment of the organism. This model of mitochondrial disease allows the generation of several predictions, testable using currently available laboratory techniques. Avenues of potential therapeutic value are indicated, including the avoidance of hypoglycaemia and the use of selective mitochondrial toxins.
\end{abstract}

There has been much recent interest in the mitochondrial diseases such as Kearns-Sayre syndrome and Leber's optic neuropathy. This has developed in parallel with sensitive assays of mitochondrial function, especially of the complexes of the respiratory chain. It has been shown that the mitochondrial DNA (mtDNA) of some patients with mitochondrial myopathy $(\mathrm{MtM})$ has suffered substantial deletions ${ }^{12}$ or duplications. ${ }^{3}$ The abnormal mtDNA is found

Institute of Medical Genetics, University of Wales College of Medicine, Heath Park, Cardiff CF4 4XN. A Clarke

Received for publication 17 May 1988.

Revised version accepted for publication 22 February 1990. principally in the mitochondria of muscle, and to a lesser extent in the mitochondria of leucocytes or fibroblasts, and only affects a subpopulation of the muscle mitochondria in each case. It has been suggested that the defective mitochondria would be subject to negative selection in rapidly dividing cell lineages, such as bone marrow stem cells, and would therefore not be found in such tissues. ${ }^{1}$ In contrast, relatively stable tissues, such as muscle, would not benefit from this selection and would therefore be subject to mitochondrial disease (MtD). Another suggestion, relevant to the benign, reversible cytochrome c oxidase deficient mitochondrial myopathy ${ }^{4}$ only, is that improvement occurs as a result of the impaired viability of muscle fibres containing large numbers of defective mitochondria. ${ }^{5}$ Negative selection against mitochondria with deleted genomes may be more intense than it is against those with duplications. ${ }^{3}$

This paper discusses recent clinical and genetic studies of mitochondria and mitochondrial disease, and proposes an explanation of the clinical features of those cases of MtD produced by defects of the mtDNA. This hypothesis does not apply to those mitochondrial disorders caused by defects in the nuclear genome. However, this model accounts for the tissue distribution of $\mathrm{MtD}$, the great clinical variation of disease both within and between families, and the evolutionary tendency for mitochondrial genes to be transferred to the nuclear genome. In addition, several predictions are made that are potentially falsifiable.

\section{Disease model}

The central hypothesis is that some defects of mtDNA give a selective advantage to their mitochondrial lineage. It has been clear for many years that defective mitochondria accumulate in the MtMs, to produce the 'ragged red fibres': that they accumulate selectively in the fibres with the lowest cytochrome c oxidase activity, in this class of myopathy, has also been known for some time. ${ }^{6}$ Indeed, 
morphological changes in cases of MtM suggest unusually active mitochondrial replication by budding. ${ }^{7}$ Protein synthesis is increased up to tenfold in the defective mitochondria of Kearns-Sayre syndrome patients who lack a specific product of mitochondrial translation. ${ }^{8}$ It is probable that mitochondria are subject to feedback control of protein synthesis in general, and of their own replication in particular, in accordance with the metabolic state of the cell/tissue. Muscle tissue is regularly subject to periods of relative ischaemia and of anaerobic metabolism, and a region within a muscle fibre that contained a focus of defective mitochondria would suffer a more prolonged and more severe local accumulation of metabolites such as lactic acid, and a corresponding paucity of ATP and phosphocreatine. This is supported by the finding of an increased density of capillaries specifically around the 'ragged red fibres' in some MtDs. ${ }^{9}$

In these circumstances, the defective mitochondria would be stimulated to replicate, and they would spread throughout the fibre. Particularly if this occurred during a period of growth, the selective replication of defective mitochondria could swamp the population of healthy mitochondria originally present in the same fibre. This would produce a myopathy, but only if the mtDNA defect impaired mitochondrial metabolism without preventing DNA synthesis and mitochondrial replication. Such a situation could arise by fresh mutation at any time, but would probably have to be present in a proportion of the mitochondria of the ovum for major adverse consequences in the individual person. It may be that defective mitochondria will only replicate rapidly in the presence of intact mitochondria, capable of supporting the protein synthesis and replication of their neighbours: this could result in a stable proportion of defective mitochondria that could be supported in a tissue under a given set of circumstances. This would be a balance between the need for healthy mitochondria to permit replication, and the drive for replication of the defective organelles. No selective advantage would arise for defects caused by mutations in the nuclear genome, because any such defect would affect all mitochondria-at least, all mitochondria within a tissue.

If there are few healthy mitochondria in an ovum, then the zygote is unlikely to survive. However, if only a small subpopulation is defective, then embryonic and fetal development can proceed. Several factors will determine the clinical consequences of defective mitochondria in an individual person.

(1) Chance: which tissues contain defective mitochondria ab initio. If the defective mitochondria in an ovum are clustered, as is possible, then the tissues corresponding to that region of the ovum are more likely to be affected. ${ }^{10}$

(2) The nature of the defect: this will determine whether or not the defective mitochondria are capable of replication, and what selective advantage is possible under different circumstances.

(3) Positive selection: there are several circumstances in which defective mitochondria could be at a Darwinian advantage.

(i) Muscle tissue: is regularly subject to metabolic stress that must be accentuated in areas with defective mitochondria. This is true whether the muscle is using glycogen or fatty acids. Tissues with a blood supply sufficient to prevent anaerobic metabolism under usual circumstances will be less prone to mitochondrial disease.

(ii) Central nervous system: requires functioning mitochondria to use the non-glucose fuels such as ketone bodies that are especially important in infancy and during periods of fasting. Defective mitochondria will therefore be at a replicative advantage during infancy, and also during spells of relative hypoglycaemia/fasting later in life, and will predispose to CNS dysfunction. The localised lactic acidosis that would be maximal in certain areas within the brain could also cause transient dysfunction or permanent damage from cell necrosis, and hence produce the MELAS (mitochondrial encephalopathy, lactic acidosis, and stroke-like episodes) or MERRF (myoclonus, epilepsy, and ragged red fibres) syndromes. ${ }^{5} 11$ Vascular smooth muscle involvement in the MtD may also contribute to the MELAS symptomatology. ${ }^{12} \mathrm{~A}$ similar explanation may account for the retinal involvement in some cases of mitochondrial disease, and for the apparently sudden onset of some mitochondrial disorders (for example, Leber's optic atrophy).

(iii) Fetal life: with birth comes the switch to fully aerobic metabolism in most tissues. Until then, the $\mathrm{pH}$ and $\mathrm{Po}_{2}$ are both relatively low. It is possible that a mitochondrial defect could enjoy a selective advantage in fetal life which is lost from the time of birth. This hypothesis could account for cases of MtD that present with severe neonatal myopathy but then recover if the child lives through the first few weeks or months. ${ }^{4}$ The intact mitochondria are at a replicative advantage during this time, and displace the defective ones.

There is another hypothesis, that mitochondria normally use a succession of different nucleus encoded isozymes, as with the succession of $\beta$ haemoglobins in erythrocytes, and that these cases represent a defect of the 'fetal' form of a nuclear component of the respiratory chain. Such a mechanism could well account for this condition when the defect is in the nuclear genome. To my knowledge, however, no such cases have been found in humans, whereas defects of mitochondrially encoded proteins have been found (reference 13: especially the fifth, sixth, and seventh cases).

(4) Syncytia: in a tissue where cells form syncytia, 
any replicative advantage enjoyed by defective mitochondria could result in a massive dissemination of the defect and a high proportion of the tissue being affected. Cardiac and skeletal muscle are both put at risk of MtM in this way. Cellular tissues with little mitotic activity after normal growth is complete, for example, kidney and liver, will not suffer from $\mathrm{MtD}$ even if they initially contain the same proportion of defective mitochondria. There is not the same metabolic stress, and the cell membrane limits any possible spread of the disorder.

(5) Embryonic origin and bulk of tissue: a small muscle derived from only a few embryonic cells will have a smaller chance of containing defective mitochondria than a large muscle. However, if embryonic cells destined to generate an external ocular muscle happen to contain defective mitochondria, and these are favoured by selection, then this is much more likely to cause symptoms than if the same number of fibres were defective in vastus lateralis. The predilection of MtM for the oculomotor muscles and levator palpebrae superioris can be explained in this way. Similarly, specialised tissues consisting of few cells, such as the cardiac conduction tissue, will be at risk of $\mathrm{MtD}$. This may account for the association of endocrine disease with mitochondrial disease, because endocrine tissue bulk is small, and each type of secretory cell derives from only a few cells in the embryo.

(6) Redundancy of cellular function: if a fraction of cells in liver or kidney functions suboptimally, the organ can compensate up to quite generous limits. The same is not true for the central and peripheral nervous systems and for cardiac conduction tissue. Defective Purkinje fibres and peripheral neurones can cause problems at any age. Failure of specific regions of the CNS after plasticity has been lost will also cause problems corresponding to the function that was served by the malfunctioning cell or clone of cells (which are likely to share both the same mitochondrial defect and much the same function within the CNS). This explains why some tissues are especially susceptible to MtDs.

\section{Discussion}

It can be seen that a defect in mtDNA could have a selective advantage, could achieve dominance within a tissue, and cause disease. This is particularly so if the tissue is syncytial, is derived from a few embryonic cells only, has little redundancy of function, and is subject to repeated or continuous metabolic stress. That MtM affects skeletal and cardiac muscle, cardiac conduction tissue, and the peripheral and central nervous systems is comprehensible. Each affected family will have a distinct mutation/deletion of the mtDNA in its defective population of mitochondria, so the clinical pattern will vary with the defect in each case. In addition, random processes will determine the distribution of normal and defective mitochondria within the ovum and hence within the embryo. This will result in considerable variation among the affected members of a family. ${ }^{14}$ Others have suggested that the tissue specificity of $\mathrm{MtD}$ is the result of defects in tissue specific, nuclear encoded proteins. ${ }^{15} 16$ Such defects may exist, but this explanation will not so easily account for cases with known defects in the mitochondrial genome. The possibility that mitochondrial selection may contribute to the clinical course of MtD has been advanced, ${ }^{13}$ but not advocated. The present paper elaborates that suggestion and examines the testable consequences. One important item of evidence in favour of this model is the finding that even a single mitochondrion, if its genome differs from the others in the same cell, can exploit a selective advantage in tissue culture to replace the original mitochondrial population of the cell. ${ }^{17}$

A number of cases of MtD are known in which several complexes of the respiratory chain are defective, or in which several RNA or polypeptide chains are lacking. ${ }^{1218}$ One explanation is that the mtDNA could be deleted for several genes encoding the relevant protein subunits; or possibly tRNA genes could be deleted, which would disrupt the synthesis of several proteins. ${ }^{12}$ About $40 \%$ of $\mathrm{MtM}$ cases have major deletions of mtDNA. ${ }^{19}$ Other possibilities, however, do exist. There could be a defect of a (nuclear encoded) structural protein required for the correct attachment and functioning of the respiratory complexes to each other or to the mitochondrial inner membrane. There could be a defect in a mitochondrial receptor for nuclear encoded, cytosol synthesised proteins in whose absence these proteins cannot enter the mitochondria. ${ }^{20}$ All these possibilities will have to be considered as we learn more about the MtDs. In cases where the mtDNA is deleted for tRNA genes, ${ }^{2}$ the question arises as to how protein synthesis procedes at all. Could these defective mitochondria be dependent upon tRNAs produced in other mitochondria, and transported through the cytoplasm or through a mitochondrial web? Such a web of mitochondria may indeed exist, at least in the central mitochondria of muscle, where they form grids around the myofibrils. ${ }^{7}$

\section{CLASSIFICATION}

The clinical classification of types of MtD is clearly inadequate, as discussed in a recent, thorough review of such cases. ${ }^{21}$ Whereas mtDNA deletions are often associated with ophthalmoplegia, the spectrum of accompanying defects is so wide and variable that a classification in such terms is far too crude to be helpful $^{22}$ : it seems that even visceral pathology can result from mtDNA deletions. ${ }^{23} \mathrm{~A}$ genetic classi- 
fication may well be appropriate for defects of nucleus encoded genes, since there is likely to be good clinicalmolecular correlation. For mtDNA defects, however, there is likely to be only poor clinical-DNA correlation. A dual system of classifying these disorders will be necessary, incorporating both the site, nature, and tissue distribution of the molecular defect, and the clinical presentation.

The one example of good DNA-clinical correlation that might be used to oppose my statement is the case of Leber's optic neuropathy, associated with a single base change at nucleotide $11778 .^{24}$ However, this mutation accounts for only a proportion of Leber cases, ${ }^{25}$ and the disorder is in any case highly variable even within families, manifesting as a generalised encephalopathy, a movement disorder, cardiac conduction defects, or optic neuropathy. The finding of a biochemical abnormality in the mitochondrial electron transport chain of affected persons ${ }^{26}$ is compatible with the intracellular selection model of MtD proposed here.

The accumulation of mtDNA mutations in muscle and other tissues with advancing age has been proposed as an important contributor to ageing and degenerative disease. ${ }^{27}$ Certainly, mitochondrial function has been shown to decline with age in 'healthy' subjects. ${ }^{28}$ While the advocates of this hypothesis cite the high mutation rate in mtDNA and the lack of a repair system as supporting evidence, I would add that positive selection in favour of new mutation mtDNA defects could occur, rather than their passive accumulation and random segregation between cells. This would naturally accelerate the process of senescence: perhaps mega-doses of ascorbic acid will prove to be beneficial after all!

\section{EVOLUTION}

Mitochondria and chloroplasts have most probably evolved from prokaryotic endosymbionts of the early eukaryotes. This helps to explain the resemblance of the transcription and translation systems of these organelles to those of bacteria. The mitochondrial genome of unicellular eukaryotes is larger, and codes for more RNA and protein species than does the mitochondrial genome of man. Thus, yeast mtDNA $(78 \mathrm{~kb})$ includes several genes for tRNAs and proteins (some as yet unidentified) that are not found in the smaller $(17 \mathrm{~kb})$ human mtDNA. The explanation of this is that the short and long term interests of the yeast mitochondria are identical to those of the whole organism. This is not true of man: the short term interests of defective mitochonciria in man can be contrary to their own long term interests, and those of the 'host' organism (the patient with MtD).

Once multicellular organisms developed tissues whose metabolic activities differed substantially, the possibility of mitochondrial disease will have arisen.
This will have resulted in selection in favour of the transfer of many functions of the mtDNA into the nucleus. Why this process is not complete, why the mitochondrial genome still exists in man, is not clear. Perhaps the mitochondria have retained little more DNA than is required for them to coordinate their own replication, although we do not know how this is achieved. Alternatively, the existence of multiple copies of the mtDNA dispersed in the cell may be advantageous, and the slight differences between the genetic triplet codes of organelle and nucleus may have made the successful transfer of certain genes to the nucleus into an improbability.

Another evolutionary consequence of the transfer of $\mathrm{mtDNA}$ sequences to the nucleus is that populations and species will differ in the functions remaining to the mitochondrial genome. Interspecies hybrids may then be viable, because at least one copy of each gene will be present in the nucleus, but may also be infertile. This would arise if the two species' $m$ tDNAs had lost different genes to the nucleus, because many of the offspring would then inherit some such genes from neither parent. There could, on this model, be differential infertility, depending upon which species had provided the hybrid's mother. Could this effect be important in determining the infertility of interspecies hybrids?

\section{PREDICTIONS AND POSSIBLE THERAPIES}

This model of mitochondrial evolution and disease permits certain extrapolations to be made (concerning cases in which the defect is in the mtDNA).

(1) The behaviour of mitochondria in tissue culture from cases of MtM should correlate with the clinical picture. For the severe but reversible neonatal myopathy, the defective mitochondria should have a demonstrable advantage under the metabolic conditions found in utero, and this should be reversed under 'normal' metabolic conditions. The metabolic conditions required to elicit a selection in favour of defective mitochondria in other cases of MtM will probably be more stringent, representing a significant lactic acidosis, or at least a substantial perturbation of intracellular energy metabolism. Such defects should be demonstrable in the myoblasts found in tissue culture of muscle from cases of MtM. Skin fibroblasts from cases of lactic acidosis in infancy have been found to have defects of the mitochondrial respiratory chain $^{29}$ : it might be possible to seek evidence of mitochondrial selection in fibroblasts from these patients as well as in muscle tissue. It may also be possible to use the technique of King and Attardi ${ }^{17}$ for demonstrating mitochondrial selection with mitochondria from cases of MtD injected into human cells in culture. Their demonstration in vitro that exogenous mitochondria can repopulate human cells lacking mtDNA has established a methodology that 
could address the issues raised in this paper, and could be used to test the possible efficacy of potential therapies for patient specific mtDNA defects. ${ }^{30}$

These techniques will provide an understanding of how the replication of mitochondria in various $\mathrm{MtDs}$ is influenced by the surrounding metabolic milieu, and may provide a means to examine the normal control of mitochondrial replication within different tissues of the higher organisms. There have already been suggestions that variation in mitochondrial function may be partitioned into additive effects of the nucleus and the mtDNA, and into an element accounted for by nucleus-mtDNA interactions.

(2) Another prediction would be that the severe, reversible, neonatal MtM should particularly affect muscles whose blood supply derives from the descending aorta more severely than muscles of the right upper limb and the brain, because these tissues are privileged in utero through receiving blood of higher oxygen content than elsewhere. The defective mitochondria should be less evident in these tissues. This effect could be sought with nuclear magnetic resonance spectroscopy, or through taking muscle biopsy samples from several sites.

(3) According to this model, selection in favour of the mtDNA defect in CNS and retina will be exacerbated by hypoglycaemia. It may therefore be helpful to prevent even trivial hypoglycaemia, and the associated switch to alternative fuels for the brain. To achieve this would necessitate the use of frequent starchy feeds by day and gastric drip feeds by night in children with mitochondrial disease of any sort, as in the management of glucose-6-phosphatase deficiency. Similarly, those with MtM should be encouraged to perform gentle exercise only: strenuous exertion could assist the selection of the defective mitochondria.

(4) Another therapy of possible value would be chloramphenicol: this well known mitochondrial poison may be found to discriminate against the defective mitochondria in cases of $\mathrm{MtM}$. This will have to be tested in vitro, possibly by the techniques of King and Attardi ${ }^{17}{ }^{30}$ before it is used clinically. Perhaps a continuous, low dose regimen would suppress the disease without causing a 'grey baby syndrome'. Other antimicrobial drugs, inhibitors of bacterial protein synthesis or replication, could also be considered for such use.

(5) Redox agents that will assist the tissues by circumventing the need for the full oxidative phosphorylation pathway may also be helpful, as previously suggested $^{28}$ and practised. ${ }^{31}$

Finally, this proposal provides an example of selfish DNA that could be of interest to evolutionary theorists. As with meiotic drive, the intracellular Darwinian selection of defective mitochondria allows experiments to be devised that will test our models of evolution as well as of disease.
I would like to acknowledge the helpful comments of Drs M Johnson, D Turnbull, and H S A Sherratt.

1 Holt IJ, Harding AE, Morgan-Hughes JA. Deletions of muscle mitochondrial DNA in patients with mitochondrial myopathies. Nature 1988;331:717-9.

2 Lestienne P, Ponsot G. Kearns-Sayre syndrome with muscle mitochondrial DNA deletion. Lancet 1988;i:885.

3 Poulton J, Deadman ME, Gardiner RM. Duplications of mitochondrial DNA in mitochondrial myopathy. Lancet 1989; 236-40.

4 DiMauro S, Nicholson JF, Hays AP, et al. Benign infantile mitochondrial myopathy due to reversible cytochrome c oxidase deficiency. Ann Neurol 1983;14:226-34.

5 DiMauro S, Bonilla E, Zeviani M, et al. Mitochondrial mvopathies. Ann Neurol 1985;17:521-38.

6 Johnson MA, Turnbull DM, Dick DJ, Sherratt HSA. A partial deficiency of cytochrome $c$ oxidase in chronic progressive external ophthalmoplegia. $\mathcal{F}$ Neurol $S_{c i}$ 1983;60:31-53.

7 Stadhouders AM, Sengers RCA. Morphological observations in skeletal muscle from patients with a mitochondrial myopathy. f Inher Metab Dis 1987;10 (suppl 1):62-80.

8 Byrne E, Marzuki S, Sattayasai N, et al. Mitochondrial studies in Kearns-Sayre syndrome: normal respiratory chain function with absence of a mitochondrial translation product. Neurolog (Minneap) 1987;37:1530-4.

9 Carpenter S, Karpati G, Heller I. Inclusion body myositis: a distinct variety of idiopathic inflammatory myopathy. Neurolog (Minneap) 1978;28:8-17.

10 Schapira AHV. Mitochondrial myopathies. Br Med J 1989;298 $1127-8$.

11 Pavlakis SG, Phillips PC, DiMauro S, De Vivo DC, Rowland LP. Mitochondrial myopathy, encephalopathy, lactic acidosis, and stroke-like episodes: a distinctive clinical syndrome. Ann Neurol 1984;16:481-8.

12 Sakuta $R$, Nonaka I. Vascular involvement in mitochondrial myopathy. Ann Neurol 1989;26:594-601.

13 Bolhuis PA, Barth PG, Wijburg FA, et al. Molecular basis of mitochondrial myopathies. Lancet 1988; i:884.

14 Ishitsu T, Miike T, Kitano A, et al. Heterogeneous phenotypes of mitochondrial encephalomyopathy in a single kindred. Neuro$\log$ (Minneap) 1987;37:1867-9.

15 Tager JM. Inborn errors of cellular organelles: an overview. F Inher Metab Dis 1987;10 (suppl 1):3-10.

16 Kroon AM, van den Bogert C. Biogenesis of mitochondria and genetics of mitochondrial defects. F Inher Metab Dis 1987;10 (suppl 1):54-61.

17 King MP, Attardi G. Injection of mitochondria into human cells leads to a rapid replacement of the endogenous mitochondrial DNA. Cell 1988;52:811-9.

18 Schapira AHV, Morgan-Hughes JA, Cleeter MJW, et al. Molecular basis of mitochondrial myopathies: polypeptide analysis in complex I deficiency. Lancet 1988;i:500-3.

19 Holt IJ, Harding AE, Cooper JM, et al. Mitochondrial myopathies: clinical and biochemical features of 30 patients with major deletions of muscle mitochondrial DNA. Ann Neurol 1989;26: 699-708.

20 Hartl FU, Schmidt B, Wachter E, Weiss H, Neupert W. Transport into mitochondria and intramitochondrial sorting of the $\mathrm{Fe} / \mathrm{S}$ protein of ubiquinol-cytochrome $\mathrm{c}$ reductase. Cell 1986;47:939-51.

21 Petty RKH, Harding AE, Morgan-Hughes JA. The clinical features of mitochondrial myopathy. Brain 1986;109:915-38.

22 Moraes CT, DiMauro S, Zeviani M, et al. Mitochondrial DNA deletions in progressive external ophthalmoplegia and Kearns-Sayre syndrome. N Engl f Med 1989;320:1293-9.

23 Rotig A, Colonna M, Bonnefont JP, et al. Mitochondrial DNA deletion in Pearson's marrow/pancreas syndrome. Lancet 1989; i:902-3.

24 Singh G, Lott MT, Wallace DC. A mitochondrial DNA mutation as a cause of Leber's hereditary optic neuropathy. $N$ Engl f Med 1989;320:1300-5.

25 Holt IJ, Miller DH, Harding AE. Genetic heterogeneity and mitochondrial DNA heteroplasmy in Leber's hereditary optic neuropathy. F Med Genet 1989;26:739-43.

26 Parker WD, Oley CA, Parks JK. A defect in mitochondrial 
electron-transport activity (NADH-coenzyme Q oxidoreductase) in Leber's hereditary optic neuropathy. $N$ Engl $f$ Med 1989:320:1331-3.

27 Linnane AW, Marzuki S, Ozawa T, Tanaka M. Mitochondrial DNA mutations as an important contributor to ageing and degenerative diseases. Luncet 1989; i:642-5.

28 Trounce I, Byrne E, Marzuki S. Decline in skeletal muscle mitochondrial respiratory chain function: possible factor in ageing. Lancet 1989;i:637--9.

29 Robinson BH, Ward J, Goodyer P, Baudet A. Respiratory chain defects in the mitochondria of cultured skin fibroblasts from three patients with lactic acidaemia. I Clin Invest 1986;77: $1422-7$

30 King MP, Attardi G. Human cells lacking mtDNA: repopulation with exogenous mitochondria by complementation. Science 1989;246:500-3

31 Ihara Y, Namba R, Kuroda S, Sato 'T, Shirabe T. Mitochondrial encephalomyopathy (MELAS): pathological study and successful therapy with coenzyme $Q_{10}$ and idebenone. $7 \mathrm{Neurol} S \mathrm{Si}$ 1989;90:263-71. 